\title{
TAYLOR SERIES OF FUNCTIONS WITH VALUES IN DUAL QUATERNION
}

\author{
Ji Eun Kim ${ }^{\mathrm{a}}$, Su Jin LIM $^{\mathrm{b}}$ AND KwANG Ho ShON ${ }^{\mathrm{c}, *}$
}

\begin{abstract}
We define an $\varepsilon$-regular function in dual quaternions. From the properties of $\varepsilon$-regular functions, we represent the Taylor series of $\varepsilon$-regular functions with values in dual quaternions.
\end{abstract}

\section{INTRODUCTION}

Fueter [2] and Naser [8] have studied properties of quaternionic differential equations as a generalization of the extended Cauchy-Riemann equations in the complex holomorphic function theory and Nôno $[9,10,11]$ has given a definition of regular functions over the quaternion field $\mathcal{T}$ identified with $\mathbb{R}^{4}$. In 1979, Sudbery [15] developed quaternionic regular function theories. By using a generalization of the Cauchy-Riemann equation, Ryan $[12,13]$ has developed regular function theories on complex Clifford algebra of quaternion valued functions.

In 1873, Clifford [1] originally conceived the algebra of dual numbers. Dual algebra has been often used for closed form solutions in the field of displacement analysis. Kotelnikov [6] and Study [14] developed dual vectors and dual quaternions for use in the application of mechanics and realized that this associative algebra was ideal for describing the group of motions of three-dimensional spaces.

In 2011, Koriyama, Mae and Nôno [5] investigated hyperholomorphic functions and holomorphic functions in quaternion analysis. In 2012, Gotô and Nôno [3] researched regular functions with values in a commutative subalgebra of matrix algebra in four real dimension and we [7] obtained regularities of functions with values in subalgebra of matrix algebras in complex $n$-dimensional.

Received by the editors July 8, 2013. Revised October 18, 2013. Accepted November 7, 2013. 2010 Mathematics Subject Classification. 32A99, 11E88, 32A10, 30 G35.

Key words and phrases. regular functions, Clifford analysis, dual quaternion, Taylor series.

*This work was supported by a 2-Year Research Grant of Pusan National University.

*Corresponding author. 
In this paper, we introduce the dual quaternion numbers and give some properties of $\varepsilon$-regular functions in dual quaternions by using the associated Pauli matrices. We give the notation of the derivative for functions with values in dual quaternions and obtain the representation of the Taylor series of $\varepsilon$-regular functions.

\section{Preliminary}

A dual quaternion is an ordered pair of quaternions and is constructed from eight base elements $e_{0}, e_{1}, e_{2}, e_{3}, \varepsilon, e_{1} \varepsilon, e_{2} \varepsilon$ and $e_{3} \varepsilon$. We consider the associated Pauli matrices

$$
e_{0}=\left(\begin{array}{ll}
1 & 0 \\
0 & 1
\end{array}\right), e_{1}=\left(\begin{array}{cc}
i & 0 \\
0 & -i
\end{array}\right), e_{2}=\left(\begin{array}{cc}
0 & 1 \\
-1 & 0
\end{array}\right), e_{3}=\left(\begin{array}{cc}
0 & i \\
i & 0
\end{array}\right),
$$

where $i=\sqrt{-1}$. And, we let the dual quaternion identity

$$
\varepsilon=\left(\begin{array}{ll}
0 & 1 \\
0 & 0
\end{array}\right)
$$

which is a nonzero and satisfy $0 \varepsilon=\varepsilon 0=0,1 \varepsilon=\varepsilon 1=\varepsilon, \varepsilon^{2}=0$ and

$$
\mathcal{D H}:=\left\{z=\zeta+\zeta^{*} \varepsilon \mid \zeta, \zeta^{*} \in \mathcal{T}\right\} \cong \mathcal{T} \times \mathcal{T},
$$

where $\zeta=\sum_{j=0}^{3} e_{j} x_{j}, \zeta^{*}=\sum_{j=0}^{3} e_{j} x_{j}^{*}$ and $x_{j}^{*}$ is a dual quaternion component of $x_{j}$ $\left(x_{j}, x_{j}^{*} \in \mathbb{R}\right)$. The element $e_{0}$ is the identity, the element $\varepsilon$ is the dual identity of $\mathcal{D H}$ and the element $e_{1}$ identifies the imaginary unit $i=\sqrt{-1}$ in the $\mathbb{C}$-field of complex numbers. We can identify $\mathcal{D} \mathcal{H}$ with $\mathbb{C}^{4}$.

The dual quaternionic conjugation $z^{*}$ of $z$, the absolute value $|z|$ of $z$ and an inverse $z^{-1}$ of $z$ in $\mathcal{D H}$ are defined, respectively, by

$$
\begin{aligned}
z^{*} & =\bar{\zeta}+\overline{\zeta^{*}} \varepsilon \\
|z|^{2} & =z z^{*}=\sum_{j=0}^{3}\left(x_{j}+\varepsilon x_{j}^{*}\right)^{2}, \\
z^{-1} & =\frac{z^{*}}{|z|^{2}}
\end{aligned}
$$

where $\bar{\zeta}=\sum_{j=0}^{3} \overline{e_{j}} x_{j}, \overline{\zeta^{*}}=\sum_{j=0}^{3} \overline{e_{j}} x_{j}^{*}$ and $\overline{e_{0}}=e_{0}, \overline{e_{j}}=-e_{j}(j=1,2,3)$.

Let $\Omega$ be an open subset of $\mathbb{C}^{2} \times \mathbb{C}^{2}$ and the dual quaternion function

$$
f: \Omega \longrightarrow \mathcal{D H}
$$

satisfy 


$$
z \in \Omega \longmapsto f(z)=\sum_{j=0}^{3} e_{j} f_{j}\left(\zeta, \zeta^{*}\right) \in \mathcal{D} \mathcal{H},
$$

where $f_{j}\left(\zeta, \zeta^{*}\right)=u_{j}\left(\zeta, \zeta^{*}\right)+\varepsilon u_{j}^{*}\left(\zeta, \zeta^{*}\right)$ and $u_{j}, u_{j}^{*}(j=0,1,2,3)$ are real-valued functions.

We use the following two dual quaternion differential operators which are defined as

$$
D:=\frac{\partial}{\partial \zeta}+\frac{\partial}{\partial \zeta^{*}} \varepsilon \text { and } D^{*}=\frac{\partial}{\partial \bar{\zeta}}+\frac{\partial}{\partial \bar{\zeta}^{*}} \varepsilon
$$

where

$$
\frac{\partial}{\partial \zeta}=\sum_{j=0}^{3} \overline{e_{j}} \frac{\partial}{\partial x_{j}}, \frac{\partial}{\partial \zeta^{*}}=\sum_{j=0}^{3} \overline{e_{j}} \frac{\partial}{\partial x_{j}^{*}}, \frac{\partial}{\partial \bar{\zeta}}=\sum_{j=0}^{3} e_{j} \frac{\partial}{\partial x_{j}}, \frac{\partial}{\partial \overline{\zeta^{*}}}=\sum_{j=0}^{3} e_{j} \frac{\partial}{\partial x_{j}^{*}} .
$$

Then we have

$$
\begin{aligned}
D^{*} f= & \left(\frac{\partial}{\partial \bar{\zeta}}+\frac{\partial}{\partial \overline{\zeta^{*}}} \varepsilon\right) f \\
= & \left(\begin{array}{cc}
\frac{\partial}{\partial x_{0}}+i \frac{\partial}{\partial x_{1}} & \frac{\partial}{\partial x_{2}}+i \frac{\partial}{\partial x_{3}}+\frac{\partial}{\partial x_{0}^{*}}+i \frac{\partial}{\partial x_{1}^{*}} \\
-\frac{\partial}{\partial x_{2}}+i \frac{\partial}{\partial x_{3}} & \frac{\partial}{\partial x_{0}}-i \frac{\partial}{\partial x_{1}}-\frac{\partial}{\partial x_{2}^{*}}+i \frac{\partial}{\partial x_{3}^{*}}
\end{array}\right) \\
& \cdot\left(\begin{array}{cc}
u_{0}+i u_{1} & u_{2}+i u_{3}+u_{0}^{*}+i u_{1}^{*} \\
-u_{2}+i u_{3} & u_{0}-i u_{1}-u_{2}^{*}+i u_{3}^{*}
\end{array}\right) \\
= & \left(\begin{array}{cc}
a_{1} & a_{2} \\
a_{3} & a_{4}
\end{array}\right)
\end{aligned}
$$

where

$$
\begin{aligned}
a_{1}= & \frac{\partial u_{0}}{\partial x_{0}}-\frac{\partial u_{1}}{\partial x_{1}}-\frac{\partial u_{2}}{\partial x_{2}}-\frac{\partial u_{3}}{\partial x_{3}}-\frac{\partial u_{2}}{\partial x_{0}^{*}}-\frac{\partial u_{3}}{\partial x_{1}^{*}} \\
& +i\left(\frac{\partial u_{1}}{\partial x_{0}}+\frac{\partial u_{0}}{\partial x_{1}}+\frac{\partial u_{3}}{\partial x_{2}}-\frac{\partial u_{2}}{\partial x_{3}}+\frac{\partial u_{3}}{\partial x_{0}^{*}}-\frac{\partial u_{2}}{\partial x_{1}^{*}}\right), \\
a_{2}= & \frac{\partial u_{2}}{\partial x_{0}}+\frac{\partial u_{0}^{*}}{\partial x_{0}}-\frac{\partial u_{3}}{\partial x_{1}}-\frac{\partial u_{1}^{*}}{\partial x_{1}}+\frac{\partial u_{0}}{\partial x_{2}}-\frac{\partial u_{2}^{*}}{\partial x_{2}}+\frac{\partial u_{1}}{\partial x_{3}}-\frac{\partial u_{3}^{*}}{\partial x_{3}}+\frac{\partial u_{0}}{\partial x_{0}^{*}}-\frac{\partial u_{2}^{*}}{\partial x_{0}^{*}} \\
& +\frac{\partial u_{1}}{\partial x_{1}^{*}}-\frac{\partial u_{3}^{*}}{\partial x_{1}^{*}}+i\left(\frac{\partial u_{3}}{\partial x_{0}}+\frac{\partial u_{1}^{*}}{\partial x_{0}}+\frac{\partial u_{2}}{\partial x_{1}}+\frac{\partial u_{0}^{*}}{\partial x_{1}}-\frac{\partial u_{1}}{\partial x_{2}}+\frac{\partial u_{3}^{*}}{\partial x_{2}}+\frac{\partial u_{0}}{\partial x_{3}}-\frac{\partial u_{2}^{*}}{\partial x_{3}}\right. \\
& \left.-\frac{\partial u_{1}}{\partial x_{0}^{*}}+\frac{\partial u_{3}^{*}}{\partial x_{0}^{*}}+\frac{\partial u_{0}}{\partial x_{1}^{*}}-\frac{\partial u_{2}^{*}}{\partial x_{1}^{*}}\right),
\end{aligned}
$$




$$
\begin{aligned}
a_{3}= & -\frac{\partial u_{0}}{\partial x_{2}}-\frac{\partial u_{1}}{\partial x_{3}}-\frac{\partial u_{2}}{\partial x_{0}}+\frac{\partial u_{3}}{\partial x_{1}}+\frac{\partial u_{2}}{\partial x_{2}^{*}}-\frac{\partial u_{3}}{\partial x_{3}^{*}} \\
& +i\left(-\frac{\partial u_{1}}{\partial x_{2}}+\frac{\partial u_{0}}{\partial x_{3}}+\frac{\partial u_{3}}{\partial x_{0}}+\frac{\partial u_{2}}{\partial x_{1}}-\frac{\partial u_{3}}{\partial x_{2}^{*}}-\frac{\partial u_{2}}{\partial x_{3}^{*}}\right), \\
a_{4}= & -\frac{\partial u_{2}}{\partial x_{2}}-\frac{\partial u_{0}^{*}}{\partial x_{2}}-\frac{\partial u_{3}}{\partial x_{3}}-\frac{\partial u_{1}^{*}}{\partial x_{3}}+\frac{\partial u_{0}}{\partial x_{0}}-\frac{\partial u_{2}^{*}}{\partial x_{0}}-\frac{\partial u_{1}}{\partial x_{1}}+\frac{\partial u_{3}^{*}}{\partial x_{1}}-\frac{\partial u_{0}}{\partial x_{2}^{*}}+\frac{\partial u_{2}^{*}}{\partial x_{2}^{*}} \\
& +\frac{\partial u_{1}}{\partial x_{3}^{*}}-\frac{\partial u_{3}^{*}}{\partial x_{3}^{*}}-i\left(\frac{\partial u_{3}}{\partial x_{2}}+\frac{\partial u_{1}^{*}}{\partial x_{2}}-\frac{\partial u_{2}}{\partial x_{3}}-\frac{\partial u_{0}^{*}}{\partial x_{3}}+\frac{\partial u_{1}}{\partial x_{0}}-\frac{\partial u_{3}^{*}}{\partial x_{0}}+\frac{\partial u_{0}}{\partial x_{1}}-\frac{\partial u_{2}^{*}}{\partial x_{1}}\right. \\
& \left.-\frac{\partial u_{1}}{\partial x_{2}^{*}}+\frac{\partial u_{3}^{*}}{\partial x_{2}^{*}} \frac{\partial u_{0}}{\partial x_{3}^{*}}+\frac{\partial u_{2}^{*}}{\partial x_{3}^{*}}\right) .
\end{aligned}
$$

Definition 2.1. Let $\Omega$ be an open set in $\mathbb{C}^{2} \times \mathbb{C}^{2}$. A function $f(z)$ is said to be $\varepsilon$-regular in $\Omega$ if the following two conditions are satisfied:

(a) $f_{j}(j=0,1,2,3)$ are continuously differential functions in $\Omega$, and

(b) $D^{*} f(z)=0$ in $\Omega$.

\section{Taylor Series of Dual Quaternion Functions}

We define the derivative $f^{\prime}(z)$ of $f(z)$ by the following:

$$
f^{\prime}(z):=D f(z) \text {. }
$$

Lemma 3.1. Let $\Omega$ be a domain in $\mathbb{C}^{2} \times \mathbb{C}^{2}$ and $f(z)$ be a holomorphic mapping and $\varepsilon$-regular defined in $\Omega$. Then,

$$
\begin{aligned}
f^{\prime}(z) & =\left(\frac{\partial}{\partial \zeta}+\frac{\partial}{\partial \bar{\zeta}}+\varepsilon\left(\frac{\partial}{\partial \zeta^{*}}+\frac{\partial}{\partial \overline{\zeta^{*}}}\right)\right) f \\
& =2\left(\frac{\partial}{\partial x_{0}}+\varepsilon \frac{\partial}{\partial x_{0}^{*}}\right) f \\
& =-2 \sum_{j=1}^{3} e_{j}\left(\frac{\partial}{\partial x_{j}}+\varepsilon \frac{\partial}{\partial x_{j}^{*}}\right) f .
\end{aligned}
$$

Proof. Since $f(z)$ is an $\varepsilon$-regular function in $\Omega$, we have

$$
\begin{aligned}
f^{\prime}(z)= & \left(\begin{array}{cc}
\frac{\partial}{\partial x_{0}}-i \frac{\partial}{\partial x_{1}} & -\frac{\partial}{\partial x_{2}}-i \frac{\partial}{\partial x_{3}}+\frac{\partial}{\partial x_{0}^{*}}-i \frac{\partial}{\partial x_{1}^{*}} \\
\frac{\partial}{\partial x_{2}}-i \frac{\partial}{\partial x_{3}} & \frac{\partial}{\partial x_{0}}+i \frac{\partial}{\partial x_{1}}+\frac{\partial}{\partial x_{2}^{*}}-i \frac{\partial}{\partial x_{3}^{*}}
\end{array}\right) \\
& \cdot\left(\begin{array}{cc}
u_{0}+i u_{1} & u_{2}+i u_{3}+u_{0}^{*}+i u_{1}^{*} \\
-u_{2}+i u_{3} & u_{0}-i u_{1}-u_{2}^{*}+i u_{3}^{*}
\end{array}\right)
\end{aligned}
$$




$$
\begin{aligned}
& =\left(\begin{array}{ll}
d_{1} & d_{2} \\
d_{3} & d_{4}
\end{array}\right) \\
& =2\left(\frac{\partial}{\partial x_{0}}+\varepsilon \frac{\partial}{\partial x_{0}^{*}}\right) f \\
& =-2 \sum_{j=1}^{3} e_{j}\left(\frac{\partial}{\partial x_{j}}+\varepsilon \frac{\partial}{\partial x_{j}^{*}}\right) f,
\end{aligned}
$$

where

$$
\begin{aligned}
& d_{1}=2\left(\left(\frac{\partial u_{0}}{\partial x_{0}}-\frac{\partial u_{2}}{\partial x_{0}^{*}}\right)+i\left(\frac{\partial u_{1}}{\partial x_{0}}+\frac{\partial u_{3}}{\partial x_{0}^{*}}\right)\right) \\
& d_{2}=2\left(\left(\frac{\partial u_{2}}{\partial x_{0}}+\frac{\partial u_{0}^{*}}{\partial x_{0}}+\frac{\partial u_{0}}{\partial x_{0}^{*}}-\frac{\partial u_{2}^{*}}{\partial x_{0}^{*}}\right)+i\left(\frac{\partial u_{3}}{\partial x_{0}}+\frac{\partial u_{1}^{*}}{\partial x_{0}}-\frac{\partial u_{1}}{\partial x_{0}^{*}}+\frac{\partial u_{3}^{*}}{\partial x_{0}^{*}}\right)\right) \\
& d_{3}=-\frac{\partial u_{2}}{\partial x_{0}}+i \frac{\partial u_{3}}{\partial x_{0}} \\
& d_{4}=2\left(\left(\frac{\partial u_{0}}{\partial x_{0}}-\frac{\partial u_{2}^{*}}{\partial x_{0}}\right)+i\left(-\frac{\partial u_{1}}{\partial x_{0}}+\frac{\partial u_{3}^{*}}{\partial x_{0}}\right)\right) .
\end{aligned}
$$

From

we can get

$$
\frac{\partial}{\partial \zeta}+\frac{\partial}{\partial \bar{\zeta}}=2 \frac{\partial}{\partial x_{0}} \text { and } \frac{\partial}{\partial \zeta^{*}}+\frac{\partial}{\partial \overline{\zeta^{*}}}=2 \frac{\partial}{\partial x_{0}^{*}}
$$

$$
f^{\prime}(z)=\left(\frac{\partial}{\partial \zeta}+\frac{\partial}{\partial \bar{\zeta}}+\varepsilon\left(\frac{\partial}{\partial \zeta^{*}}+\frac{\partial}{\partial \overline{\zeta^{*}}}\right)\right) f
$$

Theorem 3.2. Let $f(z)$ be a homogeneous polynomial of degree $m$ with respect to the variables $\zeta$ and $\zeta^{*}$. If $f(z)$ is a holomorphic and $\varepsilon$-regular function in $\mathbb{C}^{2} \times \mathbb{C}^{2}$, then we have

$$
f(z)=\frac{1}{m !} f^{(m)}(z) z^{m}
$$

Proof. Since $f(z)$ is a homogeneous polynomial, we have

$$
\begin{aligned}
f(z) & =\sum_{k=0}^{m}\left(\begin{array}{c}
m \\
k
\end{array}\right) \zeta^{m-k}\left(\varepsilon \zeta^{*}\right)^{k} \\
& =\zeta^{m}+\varepsilon m \zeta^{m-1} \zeta^{*} .
\end{aligned}
$$

Then

$$
\begin{aligned}
f^{\prime}(z) & =m \zeta^{m-1}+\varepsilon m(m-1) \zeta^{m-2} \zeta^{*}, \\
f^{\prime}(z) z & =m \zeta^{m}+\varepsilon m^{2} \zeta^{m-1} \zeta^{*} .
\end{aligned}
$$


Thus, $f(z)=\frac{1}{m} f^{\prime}(z) z$. And

$$
\begin{aligned}
f^{\prime \prime}(z) & =m(m-1) \zeta^{m-2}+\varepsilon m(m-1)(m-2) \zeta^{m-3} \zeta^{*} \\
f^{\prime \prime}(z) z & =m(m-1) \zeta^{m-1}+\varepsilon m(m-1)^{2} \zeta^{m-2} \zeta^{*} .
\end{aligned}
$$

Thus, $f^{\prime}(z)=\frac{1}{m-1} f^{\prime \prime}(z) z$. Repeating the above calculation, we have

$$
f(z)=\frac{1}{m !} f^{(m)}(z) z^{m} .
$$

Theorem 3.3. Let $\Omega$ be a domain in $\mathbb{C}^{2} \times \mathbb{C}^{2}$. Let $f(z)$ be a holomorphic and $\varepsilon$-regular function in $\Omega$ and $\alpha \in \Omega$. Then there exists a neighborhood $U_{\alpha}$ of $\alpha$ such that

$$
f(z)=\sum_{m=0}^{\infty} C_{m} \zeta^{m}+\varepsilon\left\{C_{1}+\sum_{m=2}^{\infty} C_{m}\left((m-1) \zeta^{m-1}+\zeta^{m-2}\right)\right\} \zeta^{*},
$$

where $C_{m}=\frac{1}{m !} f^{(m)}(\alpha)$.

Proof. From substituting a dual number into Taylor series, we have

$$
\begin{aligned}
f(z) & =f(\zeta)+\frac{f^{\prime}(\zeta)}{1 !}\left(\varepsilon \zeta^{*}\right)+\frac{f^{\prime \prime}(\zeta)}{2 !}\left(\varepsilon \zeta^{*}\right)^{2}+\frac{f^{(3)}(\zeta)}{3 !}\left(\varepsilon \zeta^{*}\right)^{3}+\cdots \\
& =f(\zeta)+\varepsilon f^{\prime}(\zeta) \zeta^{*}
\end{aligned}
$$

By Theorem 3.2 and $z^{m}=\zeta^{m}+\varepsilon\left((m-1) \zeta^{m-1} \zeta^{*}+\zeta^{m-2} \zeta^{*}\right)$, we have

$$
\begin{aligned}
f(z) & =C_{0}+C_{1} z+\sum_{m=2}^{\infty} C_{m}\left\{\zeta^{m}+\varepsilon\left((m-1) \zeta^{m-1} \zeta^{*}+\zeta^{m-2} \zeta^{*}\right)\right\} \\
& =C_{0}+C_{1} \zeta+\sum_{m=2}^{\infty} C_{m} \zeta^{m}+\varepsilon\left\{C_{1} \zeta^{*}+\sum_{m=2}^{\infty} C_{m}\left((m-1) \zeta^{m-1} \zeta^{*}+\zeta^{m-2} \zeta^{*}\right)\right\} \\
& =\sum_{m=0}^{\infty} C_{m} \zeta^{m}+\varepsilon\left\{C_{1}+\sum_{m=2}^{\infty} C_{m}\left((m-1) \zeta^{m-1}+\zeta^{m-2}\right)\right\} \zeta^{*}
\end{aligned}
$$

Remark 3.4. Let $\Omega$ be a domain in $\mathbb{C}^{2} \times \mathbb{C}^{2}$. If $g_{0}(z)$ is a holomorphic function with value in quaternions, then there exists a function $g_{1}(z)$ with value in quaternions such that 


$$
f(z)=g_{0}(z)+\varepsilon g_{1}(z)
$$

is $\varepsilon$-regular in $\Omega$.

By the results of Kenwright [4],

$$
f(z)=f(\zeta)+\varepsilon f^{\prime}(\zeta) \zeta^{*} .
$$

We put $g_{0}(z)=f(\zeta)$, then $D g_{0}(z)=f^{\prime}(\zeta)$. Hence we put $g_{1}(z)=D g_{0}(z) \zeta^{*}$, we have

$$
f(z)=g_{0}(z)+\varepsilon g_{1}(z) .
$$

Example 3.5. Let $\Omega$ be a domain in $\mathbb{C}^{2} \times \mathbb{C}^{2}$. If $g_{0}=\sin (n \zeta)$, then $g_{0}^{\prime}=n \cos (n \zeta)$, $n \in \mathbb{Z}$. Thus, there exists a function $g_{1}=n \cos (n \zeta) \zeta^{*}$ such that

$$
f(z)=g_{0}(z)+\varepsilon g_{1}(z) .
$$

is $\varepsilon$-regular in $\Omega$.

\section{REFERENCES}

1. W.K. Clifford: Preliminary sketch of bi-quaternions. Proc. London Math. Soc. 4 (1873), 381-395.

2. R. Fueter: Die Fuktionentheorie der Defferentialgeleichungen $\triangle u=0$ und $\triangle \triangle u=0$ mit vier reellen Variablen. Comment. Math. Helv. 7 (1934), 307-330.

3. S. Gotô \& K. Nôno: Regular functions with values in a Commutative subalgebra $\mathbb{C}(\mathbb{C})$ of Matrix algebra $M(4 ; \mathbb{R})$. Bull. Fukuoka Univ. Ed. 61 part III (2012), 9-15.

4. B. Kenwright: A beginners guide to dual-quaternions: What they are, How they work, and How to use them for 3D character hierarchies. The 20th International Conf. on Computer Graphics, Visualization and Computer Vision. June 26-28 (2012), 1-10.

5. H. Koriyama, H. Mae \& K. Nôno: Hyperholomorphic Functions and Holomorphic functions in Quaternionic Analysis. Bull. Fukuoka Univ. Ed. 60 part III (2011), 1-9.

6. A.P. Kotelnikov: Screw calculus and some applications to geometry and mechanics. Annals Imperial Univ. Kazan, 1895.

7. S.J. Lim \& K.H. Shon: Regularity of functions with values in a non-commutative algebra of complex matrix algebras. Submitted in Sci. China Math. (2013).

8. M. Naser: Hyperholomorphic functions. Siberian Math. J. 12 (1971), 959-968.

9. K. Nôno: Hyperholomorphic functions of a quaternion variable. Bull. Fukuoka Univ. Ed. 32 (1983), 21-37.

10. _ C C C C _ naracterization of domains of holomorphy by the existence of hyper-conjugate harmonic functions. Rev. Roumaine Math. Pures Appl. 31 (1986), no. 2, 159-161. 
11. _ Domains of Hyperholomorphic in $\mathbb{C}^{2} \times \mathbb{C}^{2}$. Bull. Fukuoka Univ. Ed. 36 (1987), 1-9.

12. J. Ryan : Complex Clifford Analysis. Complex Variables Theory Appl. 1 (1983), 119-149.

13. _ S Special functions and relations within complex Clifford analysis I. Complex Variables Theory Appl. 2 (1983), 177-198.

14. E. Study: Geometrie der Dynamen. Teubner, Leipzig, 1903.

15. A. Sudbery: Quaternionic analysis. Math. Proc. Camb. Phil. Soc. 85 (1979), 199-225.

${ }^{a}$ Department of Mathematics, Pusan National University, Busan 609-735, Korea Email address: jeunkim@pusan.ac.kr

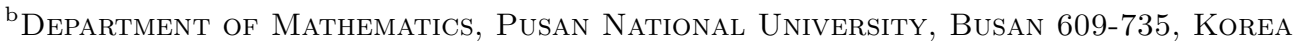
Email address: sjlim@pusan.ac.kr

${ }^{\mathrm{c}}$ Department of Mathematics, Pusan National University, Busan 609-735, Korea Email address: khshon@pusan.ac.kr 